2 Kinematics associated with treadmill walking in Rett Syndrome

3

4 Charles S. Layne ${ }^{1,2,3^{*}}$, David R. Young ${ }^{1,2}$, Beom-Chan Lee ${ }^{1,2}$, Daniel G. Glaze ${ }^{4,5}$, Aloysia

5 Schwabe ${ }^{4,5}$, Bernhard Suter ${ }^{4,5}$

6

$7 \quad{ }^{1}$ Health and Human Performance, University of Houston, Houston, Texas, United States of

8 America

9

$10{ }^{2}$ Center for Neuromotor and Biomechanics Research, University of Houston, Houston, Texas,

11 United States of America

12

$13{ }^{3}$ Center for Neuro-Engineering and Cognitive Science, University of Houston, Houston, Texas,

14 United States of America

15

164 Texas Blue Bird Circle Rett Center, Texas Children's Hospital, Houston, Texas, United States

17 of America

18

$19 \quad{ }^{5}$ Baylor College of Medicine, Houston, Texas, United States of America

20

$21 *$ Corresponding author

22

23 E-mail: clayne2@uh.edu 


\section{Kinematics of Rett walking}

Abstract

Individuals with Rett syndrome suffer from severely impaired cognitive and motor performance.

27 Current movement-related therapeutic programs often include traditional physical therapy

28 activities and assisted treadmill walking routines for those patients who are ambulatory.

However, there are no quantitative reports of kinematic gait parameters obtained during treadmill walking. Here we report the results of an investigation of 17 females diagnosed with typical Rett who walked on a treadmill as speed gradually increased. The objective included characterizing lower limb kinematics, including knee and hip joint range of motions, velocities, limb asymmetries, and the variance associated with these measures. Joint kinematics were obtained using a 12 camera motion capture system and associated processing and analysis software. Stride

35 times progressively decreased as treadmill speeds increased although the range of speeds our participant could walk was quite slow: range $0.2 \mathrm{~m} / \mathrm{s}-0.5 \mathrm{~m} / \mathrm{s}$. There were significant main

37 effects of speed on sagittal knee and hip range of motions and hip velocity. There were large joint asymmetries and variance values relative to both healthy walkers and others patient populations although variance values decreased as walking speed increased. There were

40 significant correlations between joint range of motions and stride times and joint velocities and

41 stride times. The results indicate that Rett patients can adapt their kinematic gait patterns in

42 response to increasing treadmill speed but their ability to do so lies within a narrow range of 43 speeds. We suggest that treadmill training for ambulatory individuals with Rett may further 44 promote improved walking kinematics as well as overall health benefits. 
Kinematics of Rett walking

Introduction

Mutations in the gene coding for methyl-CpG-binding protein 2 (MECP2) result in the neurodevelopmental disorder Rett Syndrome (RTT). Although a relatively rare condition,

51 worldwide RTT affects approximately 1 in 10,000 live born females [1]. Seemingly normal

52 development occurs up to ages 6-18 months at which time a period of regression with loss of

53 verbal skills and social interactions, as well as both fine and gross motor skills sets in.

54 Stereotypical hand movement, breathing difficulties, apraxia, ataxia, muscle hypertonia, limb

55 rigidity and bruxism are some of the disabling symptoms commonly observed. A period of

56 stabilization ensues, but bipedal postural control and walking are severely compromised and

57 walking ability often declines further at later ages, such that ultimately less than half remain able

58 to walk.

59

60 Loss of ambulatory skills results in a number of additional physical problems such as muscle atrophy, limb contractures, decreased cardio-respiratory fitness and low overall physical fitness.

62 Suggested physical therapy for patients with RTT has included physical exercise designed to

63 increase physical fitness and to maintain walking ability. These therapies have ranged from

64 traditional physical exercises and stretching [2] guided physical activities in a multi-sensory

65 room [3], and hydrotherapy [4]. Other authors have suggested that a program incorporating walking may have a range of benefits for individuals with RTT including improved physical

67 fitness as well as positively influencing their quality of life and wellbeing [5]. 


\section{Kinematics of Rett walking}

There are several reports of those with RTT exploring the possibility of incorporating treadmill walking into their therapeutic regimen. Lotan et al. [6], explored the use of a treadmill-walking

72 program to promote both walking skills and physical fitness and reported high correlations

73 between improved walking performance and physical fitness. While promising, this study was conducted with only four girls with RTT. Three girls with RTT served as subjects for an exploratory investigation using robot-assisted walking [7] with preliminary results indicating the girls tolerated the robotic system suggesting that this might be a clinical tool that merits further

77 investigation with RTT patients. These preliminary studies, while promising, leave open the applicability of these findings to a broader spectrum of ambulatory girls with RTT.

82 indicate improvement in gait patterns after treadmill gait training. Gait training with motorized treadmills is standard practice with a variety of populations with gait disorders, including those with Parkinson's disease, cerebral palsy and stroke. Multiple investigations have documented

85 improvements in overground gait parameters with the aforementioned populations resulting from treadmill gait training [8-13]. Individuals with RTT have neurological factors that are generally

87 different than of those conditions listed immediately above, however there is no a priori reason to walking is often a component part of therapy programs for those with RTT however its efficacy is unknown.

92 Prior to exploring the efficacy of a treadmill-walking program for both improved overall

93 physical fitness and functional walking characteristics, it is important to identify some typical 


\section{Kinematics of Rett walking}

94 kinematic features associated with treadmill walking of patients with RTT. Such information is

95 necessary to evaluate any potential improvements stemming from a treadmill-walking program.

96 Although Downs and her colleagues have completed extensive efforts to develop reliable and

97 valid measures of RTT walking that can be used as clinical measures [14] currently there are no

98 reports of lower limb quantitative kinematics obtained from individuals with RTT during

99 treadmill walking.

101 An important characteristic of effective walking is the ability to adapt to different speeds.

102 Therefore, we were interested in determining if RTT patients could modify their gait to keep

103 pace with increases in the speed of the treadmill. The basic rhythmical, alternating limb pattern

104 driving locomotion has long been proposed to be the product of a network of spinal neurons that 105 require the mediation of higher order structures for the complete expression of goal-directed

106 walking. This network is commonly referred to as a central pattern generator i.e. CPG [15, 16,

107 17]. Tonic innervation of the spinal locomotion circuits is regulated by noradrenalin and

108 serotonin neurons. Without this innervation, which [18] suggests is impaired in those with RTT,

109 due to the hypofunctioning of the aminergic neurons in the brainstem, proper functioning of the

110 spinal circuit is impaired. However, input into the circuit from lower limb muscle spindles and

111 foot contact information [19] can assist in activating the circuit to generate the basic locomotor

112 pattern [20]. The well-documented toe walking exhibited by RTT patients is proposed to be an

113 adapted behavior that generates increased spindle input to the spinal circuitry and therefore

114 activates the circuit [18]. This suggests that the basic spinal locomotion circuity remains

115 generally intact and can be activated with increased sensory input. 
Kinematics of Rett walking

117 Successful adaptation to increasing treadmill speed for those with RTT would indicate that the

118 neural mechanisms available to integrate the peripheral sensory information associated with

119 increased limb speed in a manner that lower limb kinematic parameters could be successfully

120 adapted to walk at a faster speed. Previous work by our group explored details of the temporal

121 features of gait of individuals with RTT during both overground and treadmill walking [21]. It

122 was reported there were increases in stance time but decreases in swing and double support time

123 when comparing treadmill to overground gait. Additionally, treadmill walking resulted in

124 decreased variance in the temporal gait parameters, indicating treadmill walking resulted in a

125 more regularized gait. The current work provides the first description of quantitative kinematic

126 data obtained during treadmill walking as the speed of the treadmill progressively increased.

\section{Methods}

129 Study Participants

130 Seventeen females diagnosed with typical RTT based upon the Neul et al. [22] criteria and

131 carrying pathogenic MCEP2 mutation served as subjects in this study. They ranged in age from

1324 to 20 with a mean age of 10.8 , standard deviation \pm 5.3 and were receiving treatment at the

133 Blue Bird Circle Rett Center at Baylor College of Medicine in Houston, TX. All subjects were

134 able to independently walk without orthotics and none were taking medication that would be

135 expected to impact their motor control function including benzodiazepines (often used for

136 muscle tone control). All procedures were approved by the Institutional Review Boards of the

137 Baylor College of Medicine (H-35835) and the University of Houston (00000855). The parents

138 provided written informed consent for their daughters. 
Kinematics of Rett walking

140 The task involved the subjects walking on a duel-belt motorized treadmill (Bertec®) that

141 contained force plated embedded under each belt. The subjects were secured in an overhead

142 harness that eliminated any potential falls but did not provide postural support during walking.

143 Walking was initiated at $0.1 \mathrm{~m} / \mathrm{s}$ and was increased by $0.1 \mathrm{~m} / \mathrm{s}$ every 20 seconds until either the

144 parents indicated that was the maximum speed the subject could obtain or the subject began to

145 exhibit signs of discomfort such as vocalizations, hand or facial gestures. Depending upon the

146 subject's gait pattern and treadmill speed, the 20 seconds of data collection resulted in 10-14

147 strides for each treadmill speed.

Kinematic data were collected at $100 \mathrm{~Hz}$ using a Vicon ${ }^{\circledR}$ 12-camera motion capture system in combination with the plug-in gait data processing software. Reflective markers were applied

152 forces from the treadmill force plates were sampled at $1000 \mathrm{~Hz}$ and synchronized with the

153 kinematic data. Kinematic and force data were used in combination to identify heel strike and toe 154 off. Additional details regarding the data collection procedures can be obtained in Layne et al. $155[23]$.

156 Data Processing and Analysis

157 A preliminary assessment of the data revealed that all 17 subjects were able to walk between the 158 speeds of 0.2 and $0.5 \mathrm{~m} / \mathrm{s}$ therefore the decision to analyze the kinematics associated with the 159 speeds of $0.2,0.3,0.4$ and $0.5 \mathrm{~m} / \mathrm{s}$ was made. A custom MATLAB (MathWorks $®$ ) was used to 160 filter the kinematic data with a Butterworth low-pass filter with a $6 \mathrm{~Hz}$ cut-off frequency.

161 Bilateral heel strikes were detected and the data between consecutive ipsilateral heel strikes were 162 saved as individual strides for both the right and left legs. Heel strikes were identified at the 
Kinematics of Rett walking

163

164

165

166

167

168

169

170

171

172

173

174

175

176

177

178

179

180

181

182

183

184

185

minimum position of the heel marker during each gait cycle. The toe marker minimum was used in the event the subject was toe walking on particular strides. The kinematic data were then time normalized such that each stride was represented by 100 samples. The time normalized

waveforms were then amplitude normalized such that the angular value heel strike was zero degrees. For each normalized stride, sagittal plane knee and hip angles were obtained for each

treadmill speed, for each subject. Maximum and minimum angular values were obtained and used to calculate the range of motion (ROM) for each stride. After the individual joint angles were obtained, the velocity curves for each angle were calculated. Peak angular velocity for each stride and each subject were also identified.

After the above processing was completed, the limb with the greater ROM, for each joint, was identified. The data was then reorganized into the side (i.e. left or right) with the strides of the greater ROMs grouped together and those stride with lesser ROMs grouped together. Symmetry indexes (SI) between greater and lesser joint angles were computed using the following formula [24]. A SI of 0 reflects perfect symmetry between the two limbs.

$$
\text { Symmetry Index }=1-\frac{\text { Lesser Angle }}{\text { Greater Angle }}
$$

After it was determined that there were no significant differences between the joint ROM and associated peak velocities, the data from the two limbs was collapsed for further processing and analysis. The data from each variable were then averaged for each subject, at each gait speed, and group means calculated. It was found that many of variables were not normally distributed based upon the results of the Shapiro-Wilk test of normality. Therefore, Friedman tests were 


\section{Kinematics of Rett walking}

used to determine if significant differences existed between the ROMs for each joint across the four treadmill speeds. Follow up Wilcoxon tests were utilized as appropriate with a Bonferroni correction being applied. An alpha level of $\mathrm{p}<0.05$ was adopted for significance. Pearson's correlation coefficients between a joint's ROM and its velocity and between stride times and ROMs were calculated. The above procedures were also applied to the peak velocity values to determine if limb velocity changes in response to increasing treadmill speed. To determine the relationship between the various variables associated with the gait of individuals with RTT, Pearson correlations coefficients between a joint's ROM and its velocity, between stride times and ROMs and between stride times and joint velocities were calculated. Correlations were also developed between subject age and joint ROMs and velocities. Finally, correlations were developed between stride times and subject age. To assess if the variance of the dependent measures was influenced by treadmill speed, the $F$ test for equality of variance was employed.

Occasionally our subjects' feet would cross the midline and land with one foot in front of the other. Therefore, we were interested in determining the degree of knee joint motion in the horizontal plane. We applied the same processing techniques for the knee motion in the horizontal plane as those used for sagittal joint angles. Additionally, Downs et al. [5] reported minimal vertical motion of the hip during overground walking in her subjects with RTT assessed with the Actigraph GTX3 tri-axial accelerometer device. To determine if this reported lack of vertical hip motion is a common feature of RTT gait, we analyzed the motion of the pelvis in the coronal plane. Based on the literature, we identified the range (plus two standard deviations) of transverse knee motion for healthy individuals and determined which of our subjects exceeded that range. Similarly, we identified the range (minus two standard deviations) of the vertical 
Kinematics of Rett walking

209

210

211

212

213

214

215

216

217

218

219

220

221

222

223

224 225 speeds.

226

227

\section{Results}

motion of the hip and determined if any of our subjects failed to reach the degree of motion demonstrated by healthy walkers. Descriptive statistics of the number of subjects who either exceeded the healthy range of transverse knee motion or failed to display the healthy amount hip vertical motion are reported.

The primary purpose of this investigation was to determine if individuals with RTT were able to adapt their lower limb kinematics and associated stride times as treadmill speed progressively increased. Secondary considerations included exploring the prevalence of excessive knee joint motion in the horizontal plane and pelvis motion in the frontal plane.

Table 1 displays that as treadmill speed increased from 0.2 to $0.5 \mathrm{~m} / \mathrm{s}$, our subjects were able to decrease their stride times so they could continue walking. The Friedman test revealed a significant effect for speed $(\chi 2=86.698, \mathrm{p}<0.000)$. However, only three of the 17 subjects tested were able to continue walking up to the speed of $0.6 \mathrm{~m} / \mathrm{s}$. Thus, although our subjects were able to adapt to the increasing treadmill speeds, that ability was limited to a narrow range of

\begin{tabular}{|c|c|c|c|c|}
\hline \multicolumn{5}{|c|}{ Table 1- Median stride times by speed and statistical comparisons } \\
\hline Speed & Median (s) & Comparison & Z value & P values \\
\hline 0.2 & 1.43 & & \\
\hline 0.3 & 1.27 & 0.2 vs 0.3 & -4.525 & 0.000 \\
\hline 0.4 & 1.21 & 0.3 vs 0.4 & -4.505 & 0.000 \\
\hline 0.5 & 1.17 & 0.4 vs 0.5 & -4.368 & 0.000 \\
\hline
\end{tabular}

\section{Joint ROMs}


Kinematics of Rett walking

228

229

230

231

232

233

234

235

236

237

238

239

240

241

242

243

244

245

246

247

248

249

250

251

The Friedman test revealed a significant main effect of speed on sagittal knee ROM $\left(\chi^{2}=\right.$ 11.047, $\mathrm{p}<0.011)$. Follow up Wilcoxon tests indicated that the ROM between speeds 0.2 and $0.3(0.2$ median $=9.425,0.3$ median $=10.03, \mathrm{Z}=-2.812, \mathrm{p}<0.005)$ and 0.2 and 0.4 significantly different $(0.2$ median $=9.425,0.4$ median $=9.99, Z=-2.445, \mathrm{p}<0.014)$. No other comparisons reached significance (Figure 1). Comparisons between the sagittal hip ROM and treadmill speed revealed a significant main effect of speed $((\chi 2=14.012, p<0.003)$. Significant ROM differences existed between the ROM for speeds 0.2 and $0.3(0.2$ median $=10.155 .0 .3$ median $=$ 11.385, $\mathrm{Z}=-3108, \mathrm{p}<0.003)$. There were no other significant differences for the hip ROM comparisons.

Figure 1 - Median Knee (A) and Hip (B) ROM across treadmill speeds

\section{Joint Peak Velocities}

For peak sagittal knee velocities in degrees per second, the Friedman test approached significance $(\chi 2=7.238, \mathrm{p}<0.065)$. The Friedman test for peak sagittal hip velocities revealed a significant main effect of treadmill speed $\left(\chi^{2}=14.633, \mathrm{p}<0.002\right)$. There were significant increases between treadmill speeds 0.2 and $0.3(0.2$ median $=35.0,0.3$ median $=39.1, \mathrm{Z}=$ 2.711, $\mathrm{p}<0.007)$ between speeds 0.2 and $0.4(0.2$ median $=35.0,0.4$ median $=45.3, Z=-2.711$, $\mathrm{p}<0.007)$. Interestingly there was a significant decrease between speeds 0.4 and $0.5(0.4$ median $=45.0,0.5$ median $=39.5, Z=-2.744, p<0.006)$. The Friedman test for the knee velocity in the horizontal plane revealed no significant differences across the four speeds $(\chi 2=$ 4.575, $\mathrm{p}<0.206$ ). Figure 2 displays the median angular velocities across the treadmill speeds and the associated $\mathrm{R}^{2}$ values. 
Kinematics of Rett walking

252 Figure 2 - Median knee (A) and hip (B) joint angular velocity across speeds.

There were no significant changes in the SIs for the knee and hip in the sagittal plane. Figure 3 does reflect that our subject's gait was asymmetrical with all SI values being significantly greater than 0 (i.e. perfect symmetry).

Figure 3 - Symmetry index values for the sagittal plane motion knee (solid fill) and hip joints across treadmill speeds.

The Pearson correlation value between subject age and stride time was $0.46\left(\mathrm{R}^{2}=0.21\right)$ which is significant at the $\mathrm{p}<0.01$ level. Figure 4 illustrates the high correlations between the joints' ROM and their associated velocities across the treadmill speed increases.

Figure 4 - Relationships between ROM and associated angular velocities across treadmill speeds.

Table 2 displays the Pearson R coefficients of the comparison between kinematic variables, stride times and subject age.

\begin{tabular}{|l|c|c|}
\hline \multicolumn{3}{|c|}{ Table 2 - Correlation between stride times, kinematics and age } \\
\hline Pearson Correlation Coefficients (R) & S Knee & S Hip \\
\hline Joint ROM \& Stride Times & $0.38^{*}$ & $0.59^{*}$ \\
\hline Joint Angular Velocities \& Stride Times & $0.45^{*}$ & $0.60^{*}$ \\
\hline Joint ROM \& Age & 0.15 & $0.37^{*}$ \\
\hline Joint Angular Velocity and Age & 0.12 & $0.36^{*}$ \\
\hline
\end{tabular}

*Significant at $\mathrm{p}<0.01$

The $\mathrm{F}$ tests to assess potential differences in the variance associated with the joint ROM across speeds indicated that although the variance values were very high, there were no differences 
Kinematics of Rett walking

resulting from changes in treadmill speed. The same was true for the F tests comparing knee velocities across speeds. However, significant differences in variances were found for hip velocities between speeds 0.2 vs $0.4(\mathrm{~F}=2.444, \mathrm{p}<0.006), 0.2$ vs $0.5(\mathrm{~F}=3.292, \mathrm{p}<0.000)$, and 0.3 vs $0.5(\mathrm{~F}=2.169, \mathrm{p}<0.015)$. In all cases of significant $\mathrm{F}$ tests, the slower speed was always associated with the greater variances relative to the faster speed (Table 3).

\begin{tabular}{|c|c|c|c|c|}
\hline \multicolumn{5}{|c|}{ Table 3. Variance values for sagittal plane ROM, and joint velocities } \\
\hline Speed & Knee ROM & Hip ROM & Knee Velocity & Hip Velocity \\
\hline 0.2 & 25.9 & 32.0 & 0.048 & 0.071 \\
\hline 0.3 & 35.8 & 30.3 & 0.046 & 0.047 \\
\hline 0.4 & 34.8 & 23.1 & 0.043 & 0.029 \\
\hline 0.5 & 21.7 & 19.9 & 0.027 & 0.022 \\
\hline
\end{tabular}

Using a $\mathrm{ROM}$ of $10^{\circ}$ to indicate excessive motion in the transverse plane based on values obtained with healthy individuals [25,26,27], there were only 13 instances, of a possible 136 that exceeded that threshold across all speeds and the two legs. There was no systematic effect of either age or speed on knee transverse plane motion. To determine if our subjects displayed healthy pelvic motion in the coronal plane we, used a threshold of $0.7^{\circ}$ as a minimum value to indicate if there was adequate peak motion in this plane $[28,29]$. Of the 136 measures, only six values fell below the minimal threshold value and these values were confined to just two subjects. These data confirm, with very few exceptions, our subjects with RTT displayed a range of hip motion in the coronal plane associated with healthy gait. The median transverse plane knee ROMs and median peak degrees of the hip in the coronal plane across speeds are displayed in Figure 5.

Figure 5. Median ROMs for knee transverse plane motion and median peak degrees for hip coronal plane motion across treadmill speeds.

\section{Discussion}


Kinematics of Rett walking

254 In this report, we provide the first laboratory-based information regarding kinematic gait data

255 collected from females with RTT. Characterizing the kinematic parameters associated with

256 walking of patients with RTT is important to determine if pharmacological or therapeutic

257 approaches are successful. Additionally, we were interested in determining if those with RTT

258 were able to successfully adapt their gait to increasing treadmill speeds. If so, this would suggest

259 that despite abnormal kinematic parameters, neurological mechanisms remain intact to respond

260 to the sensory feedback associated with increased treadmill speed and adapt their kinematic

261 parameters accordingly.

As reported in Table 1, the subjects were able to decrease their stride times as treadmill speed

264 increased as has been demonstrated in a sample of healthy subjects [30]. However, these decreases occurred within a relatively narrow range of treadmill speeds $(0.2-0.5 \mathrm{~m} / \mathrm{s})$. To place children who averaged 5.7 years of age were asked to walk slow, they averaged $0.97 \mathrm{~m} / \mathrm{s}$ with average stride times of 0.99 seconds. Our average stride times ranged from 1.45 seconds at speed $0.2 \mathrm{~m} / \mathrm{s}$ to 1.10 seconds at speeds $0.5 \mathrm{~m} / \mathrm{s}$. The average 10.5 year old (similar to the average age

271 in this investigation) in the Lythgo study averaged $1.04 \mathrm{~m} / \mathrm{s}$ with average stride times of 1.15

272 seconds when asked to adopt a slow gait. To provide additional perspective, 9.5 year old children

273 diagnosed with spastic diplegic cerebral palsy (CP) walked at a self-selected speed $0.86 \mathrm{~m} / \mathrm{s}$ on

274 average during overground walking [32]. This value is $72 \%$ greater than the maximum speed our

275 patients walked on the treadmill. An additional study reported that 10 year old children with

276 bilateral $\mathrm{CP}$ walked at $0.83 \mathrm{~m} / \mathrm{s}$ on average while those with unilateral $\mathrm{CP}$ walked on average at 
Kinematics of Rett walking

277

278

279

280

281

282

283

284

285

286

287

288

289

290

291

292

293

294

295

296

297

298

299

$1.01 \mathrm{~m} / \mathrm{s}$ [33]. Although not particularly surprising, these comparisons between children with CP and our subjects of similar age emphasize that girls with RTT walk significantly slower than those with CP.

Despite the minimal range of slow walking speeds, our subjects did decrease their stride times such that they were able to maintain pace with the increasing treadmill speeds. This finding strongly suggests that our subjects were able to both adequately detect the sensory information indicating the treadmill speed was increasing and integrate that information to increase their lower limb velocities that resulted in significantly decreased stride times. This is consistent with Aoi et al's [19] assertion that foot contact information and muscle spindle input can activate the CPG and adjust the locomotor pattern to meet the lower limb movement demands associated with increasing treadmill speed. Consistent with the decrease in stride times are the significant increases of knee and hip ROMs and angular velocity associated with increases treadmill speed as has been reported for a large range of healthy individuals $[34,35,36]$. These significant main effects and the highly significant correlations between knee and hip ROM and their associated angular velocities (see Figure 5) also reflect our subjects' ability to modify their lower limb kinematic motion to adapt to the increasing treadmill speeds. Our data thereby suggest that our females with RTT do have intact spinal locomotion circuity that can be regulated by the sensory input generated by walking within a narrow range of walking speeds. We speculate that our subjects are unable to increase their walking speed beyond $0.5 \mathrm{~m} / \mathrm{s}$ is primarily related to their failure to maintain their attention on the walking task as well as their inability to preserve postural stability despite the safety that the harness provided. 
Kinematics of Rett walking

Although the spinal CPG may be able to produce the fundamental alternating lower limb motion necessary to walk, the associated kinematics display a large amount of variance and the relationship between the two limbs is asymmetric. These features contribute to our subject's lack of postural stability, which therefore prevents them from being able to increase their walking speed. As observed in Figures 3 and 4, our subjects had large symmetry indices and it is worth noting that there was a significant linear trend for knee flexion asymmetry to increase as treadmill speed increased $\left(\mathrm{R}^{2}=0.90\right)$. For comparative purposes, a gait study of patients with peroneal nerve palsy displayed median knee joint angular asymmetry of $20 \%$ from perfect symmetry [37] while a group of healthy subjects displayed a 3.7\% deviation from perfect symmetry [38]. In contrast, our knee joint asymmetries ranged from $26 \%$ at $0.2 \mathrm{~m} / \mathrm{s}$ to $36 \%$ at $0.5 \mathrm{~m} / \mathrm{s}$. reflecting a high degree of asymmetry.

Another notable feature of the kinematics exhibited by our subjects is the very small range of knee joint motion despite some minimal but statistically significant speed-related increases.

Consistent with our results, previous investigations have also reported minimal changes in knee motion associated with small increases in walking speed [39]. The median values ranged from 9.4 at $0.2 \mathrm{~m} / \mathrm{s}$ to 10.3 at $0.5 \mathrm{~m} / \mathrm{s}$. This minimal knee ROM can be characterized as 'stiff-knee gait' (SKG) and contributes to the slow speeds at which our subjects were able to walk. ${ }^{\circ}$. Healthy individuals when asked to walk at $0.3 \mathrm{~m} / \mathrm{s}$ on a treadmill, a speed that our subjects walked, had an average knee ROM of 46.1 and a hip ROM of 29.6 [39]. Carriero et al. [32] published data from a sample of children with spastic diplegia $\mathrm{CP}$ aged 9.5 years and reported a mean range of $41.3^{\circ}$ while an aged match sample of typically developing children displayed a ROM of $65.4^{\circ}$. Individuals post-stroke also exhibit significantly reduced knee ROM during gait 
Kinematics of Rett walking

$323[40,41]$. For example, the post-stroke subject's in Chen et al.'s [40] investigation displayed peak

324 knee flexion of $37.8^{\circ}$ with their paretic limb while healthy controls had average peak knee

325 flexion values of 61.9. Thus, even patient populations that have been characterized as displaying

326 SKG had significantly greater knee motion that ambulatory females with RTT. Concerning hip

327 ROM in the sagittal plane, Carriero's et al. study [32] reported a range of 47.1 for children with

328 CP and 49.9 for typically developing children. Again, these values are significantly greater than

329 observed in the current study.

Our sample of females with RTT have an extremely limited lower limb ROM as well as a limited

gait also display compensatory kinematic strategies, primarily hip hiking and increased

334 circumduction to ensure adequate toe clearing [40,42]. Interestingly, except in rare cases, our

335 subjects showed no tendency toward either of the traditional kinematic compensations associated

$342 \mathrm{~m} / \mathrm{s}$ our subjects exhibited signs of discomfort and the treadmill speed was immediately

343 decreased and testing discontinued. We hypothesize that, unlike those with CP or post-stroke

344 who walk faster than our subjects and demonstrate compensatory kinematic strategies, our

345 subjects with RTT were unable to modify their kinematic strategies that would enable them to 
Kinematics of Rett walking

walk at faster speeds. Possible factors that may contribute to our participants' slow gait speeds are discussed below.

There are several factors identified in the literature that are related to severely reduced lower

SKG and this is often been attributed to hyperactivity of the rectus femoris $[36,44]$. Another suggested cause of SKG is a lack of adequate push off at the ankle [45], leading to a reduced knee velocity at toe off and therefore reduced passive knee flexion [46,47]. Reduced hip joint velocity associated with weak hip flexors is also suggested to be a potential cause of SKG $[48,49]$. All of these muscle-related issues are likely to be factors in the severely reduced lower limb ROMs and contribute to slow walking speeds observed in the current study

Besides resulting in gait kinematics that significantly reduce the speed at which our subjects could walk, these kinematic patterns are energy inefficient $[40,50]$ with oxygen consumption and cost being elevated [51]. An investigation of 12 females with RTT who walked for six minutes on a treadmill, reported that energy production was low relative to healthy subjects that could result in tiredness within a few minutes of walking [43]. As observed in Figures 3 and 4, our subjects had large symmetry indices and it is worth noting that there was a significant linear

364 trend for knee flexion asymmetry to increase as treadmill speed increased $\left(\mathrm{R}^{2}=0.90\right)$. For 365 comparative purposes, a gait study of patients with peroneal nerve palsy displayed median knee joint angular asymmetry of 20\% from perfect symmetry [37], while a group of healthy subjects

367 displayed a 3.7\% deviation from perfect symmetry [38]. In contrast, our knee joint asymmetries 
Kinematics of Rett walking

Significant kinematic asymmetries during gait are part of an overall pattern of lower limb motion that is energetically inefficient and will result in a rapid rate of fatigue development.

As has previously been reported, there was a significant positive relationship between our subject's stride times and age [21]. Interestingly however, there were low (hip) and negligible (knee) relationships between age and ROM and age and angular velocity (Table 2). Conversely, there were significant positive relationships between both knee and hip ROM with stride time and angular joint velocities with stride time. This is consistent with previous reports in that speed is a greater indicator of associated kinematic gait variables than is age $[52,53]$ and this relationship appears to hold true for those with RTT.

The data from the current study provides evidence that a relatively large sample of ambulatory individuals with RTT are able to walk on the treadmill and modify their kinematic pattern such that they are able to increase their walking speed within a limited range. Despite kinematic patterns that lead to SKG, poor dynamic postural control and limited concentration on the walking task, we suggest that those with RTT would benefit from a physical activity program that includes regular bouts of treadmill walking $[3,5,21,43]$. Heart rate, cardiac vagal tone, mean arterial blood pressure and cardiac sensitivity to baroreflex, and transcutaneous partial pressures of oxygen sampled in females with RTT respond to treadmill walking in patterns that are similar to those of healthy individuals [43]. In a recent review article focused on evaluating post-stroke physical activity programs, it was reported that three studies that used a treadmill walking intervention found significant improvements in peak oxygen uptake after the intervention [54]. These findings strongly suggest that ambulatory patients with RTT can achieve improved 
Kinematics of Rett walking

392 physical fitness resulting from a walking fitness program despite the challenges they must

393 overcome.

395 Besides improved physical fitness, a second benefit of a treadmill walking program would be

396 potential improvements in gait kinematics and postural control dynamics that could result in

397 increases in walking speed. Increases in walking speed have been reported to improve gait

398 kinematics. For example, 20 post-stroke subjects were exposed to a treadmill walking protocol

399 that required them to walk as fast as possible. The results demonstrated that compared with their

400 self-selected speed, walking as fast as possible improved the symmetry between their hemi-

401 paretic and nonparetic limbs, as well as increases in knee and hip ROM [55]. Willerslev-Olsen,

402 et al. [56] reported that the benefits of daily treadmill training over one month with 16 children

403 with CP included, significant increases in speed, improved dorsiflexion during the late portion of

404 the swing phase and increase weight acceptance on the heel during early stance. The authors

405 proposed that treadmill gait training may promote plasticity in the corticospinal tract driven by

406 sensory input into the CPG and results in their observed improvements in gait. Similar results

407 following treadmill gait training were reported in patients who had incomplete spinal cord

408 injuries [57].

409

410 An important finding is that improvement in gait kinematics can be achieved by walking at less

411 than an individual's maximal speed during treadmill training [55]. Although this study was

412 completed with individuals with chronic stroke, it has direct relevance for those with RTT who

413 often struggle to sustain their maximal achievable gait speed, even during treadmill walking.

414 Beyond, improvement in physical fitness and gait parameters, regular walking has the potential 
Kinematics of Rett walking

415 to positively influence quality of life and wellbeing of those with RTT. Given the above

416 information, it is reasonable to hypothesize that ambulatory females with RTT will also benefit

417 from a treadmill gait training protocol.

418

419 In conclusion, our investigation has demonstrated that ambulatory females with RTT are able to

420 adapt their stride times and lower limb kinematics in response to increases in treadmill belt

421 speed, albeit within a very narrow range of gait speeds. Additionally, we have characterized

422 several kinematic parameters associated with RTT, including very limited knee and hip ROM

423 and significant asymmetrical motion. Despite the altered gait characteristics, we propose that a

424 treadmill walking training program can improve the overall physical fitness as well as kinematic

425 parameters, thereby improving the quality of life for those with RTT.

426

427

428

429

430

431

432

433

434

435

436

437 
Kinematics of Rett walking

441 References

442 1. Kerr AM. Early clinical signs in the Rett disorder. Neuropediatrics 1995; 26(2): 67-71.

443

444 2. Hanks SB. Motor disabilities in the Rett syndrome and physical therapy strategies. Brain Dev

$445 \quad 1990 ; 12: 157-161$.

446

447 3. Lotan M, Shapiro M. Management of young children with Rett disorder in the controlled

448 multi-sensory (Snoezelen) environment. Brain Dev. 2005; Nov;27 Suppl 1: S88-S94.

449

450 4. Bumin G, Uvanik M, Yilmaz I, Kayihan H, Topcu M. Hydrotherapy for Rett syndrome. J

$451 \quad$ Rehabil Med. 2003; 35: 44-45.

452

453 5. Downs J, Leonard H, Jacoby P, Brisco L, Baikie G, Hill K. Rett syndrome: establishing a

454 novel outcome measure for walking activity in an era of clinical trials for rare disorders. Disabil

455 Rehabil. 2015; 37: 1992-1996.

456

457 6. Lotan M, Isakov E, Merrick J. Improving functional skills and physical fitness in children

458 with Rett syndrome. J Intellect Disabil Res. 2004; 48: 730-735. 
Kinematics of Rett walking

460

461

462

463

464

465

466

467

468

469

470

471

472

473 $474 \quad 1158$.

475

476

477

478

479

480

481

482

7. Krebs HI, Peltz AR, Berkowe J, Angacin G, Cortes M, Edwards D. Robotic biomarkers in RETT Syndrome: Evaluating stiffness. 2016; 6th IEEE International Conference on Biomedical Robotics and Biomechatronics (BioRob). 2016; June, Singapore. DOI:

10.1109/BIOROB.2016.7523704

8. Baer GD, Salisbury LG, Smith MT, Pitman J, Dennis M. Treadmill training to improve mobility for people with sub-acute stroke: a phase II feasibility randomized controlled trial. Clin Rehabil. 2018; 32(2): 201-212.

9. Bello O, Sanchez JA, Lopez-Alonso V, Marquez G, Morenilla L, Castro X, et al. The effects of treadmill or overground walking training program on gait in Parkinson's disease. Gait Posture. 2013; 38: 590-595.

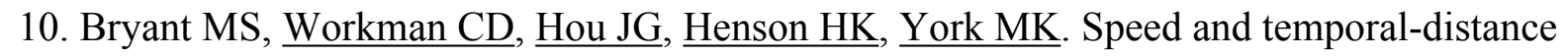
adaptations during treadmill and overground walking following stroke. PM R. 2016; 8(12): 1151-

11. Grecco LCN, Zanon L, Sampaio LMM, Oliveira, CS. A comparison of treadmill training and overground walking in ambulant children with cerebral palsy: randomized controlled clinical trial. Clin Rehabi. 2013; 27: 686-696.

12. Klamroth S, Steib S, Gaßner H, Goßler J, Winkler J, Eskofier B. et al. Immediate effects of perturbation treadmill training on gait and postural control in patients with Parkinson's disease. Gait Posture. 2016; 50: 102-108. 
Kinematics of Rett walking

484 13. Rose DK, Nadeau SE, Wu SS, Tilson JK, Dobkin BH, Pei Q, Duncan PW. Locomotor

485 training and strength and balance exercises for walking recovery after stroke: Response to

486 number of training sessions. Phys Ther. 2017; 97: 1066-1074.

487 14. Downs J, Leonard H, Wong K, Newton N, Hill K. Quantification of walking-based physical

488 activity and sedentary time in individuals with Rett syndrome. Dev Med Child Neurol. 2017 Jun;

489 59(6): 605-611.

490

491 15. Danner SM, Hofstoetter US, Freundl B, Binder H, Mayr W, Rattay F, et al., Human spinal

492 locomotor control is based on flexibly organized burst generators. Brain. 2015; 138: 577-588.

493

494 16. Grillner S. Control of locomotion in bipeds, tetrapods, and fish. In: Brooks VD, editor.

495 Handbook of physiology. Section 1: The nervous system, vol. II. Motor control. Bethesda, MD:

496 American Physiological Society. 1981: 1179-1236.

497

498 17. Haghpanah SA, Farahmand F, Zohoor H. Modular neuromuscular control of human

499 locomotion by central pattern generator. J Biomech. 2017; 53: 154-162.

500

501 18, Segawa M. Early motor disturbances in Rett syndrome and its pathophysioilogical

502 importance. Brain Dev. 2005; 27 Suppl 1:S54-S58. 
Kinematics of Rett walking

504

505

506

507

508

509

510

511

512

513

514

515

516

517

518

519

520

521

522

523

524

525

526

25. McClelland JA, Webster KE, Feller JA, Menz HB. Knee kinematics during walking at

527 different speeds in people who have undergone total knee replacement. Knee. 2011; 18: 151-155. 
Kinematics of Rett walking

529 26. Nester C. The relationship between transverse plane leg rotation and transverse plane motion 530 at the knee and hip during normal walking. Gait Posture. 2000; 12: 251-256.

531

532 27. Stief F, Böhm H, Dussa CU, Multerer C, Schwitz A, Imhoff AB, et al. Effect of lower limb 533 malalignment in the frontal plane on transverse plane mechanics during gait in young individuals 534 with varus knee alignment. Knee. 2014; 21: 688-693.

535 536

537

538

539

540

541

542

543

544

545 546 547 548 549 550 551

28. Heyrman L, Feys H, Molenaers G, Jaspers E, Monari D, Meyns P, et al. Three-dimensional head and trunk movement characteristics during gait in children with spastic diplegia. Gait Posture. 2013; 38: 770-776.

29. Molina-Rueda F, Alguacil-Diego IM, Cuesta-Gómez A, Iglesias-Giménez J, Martín-Vivaldi A, Miangolarra-Page JC. Thorax, pelvis and hip pattern in the frontal plane during walking in unilateral transtibial amputees: biomechanical analysis. Braz J Phys Ther. 2014 May-June; 18(3): 252-258.

30. Arendt-Nielsen L, Sinkjrer T, Nielsen J, Kallesøe K. Electromyographic patterns and knee joint kinematics during walking at various speeds. J Electromyogr Kinesio. 1990; 1(2): 89-95.

31. Lythogo N, Wilson C, Galea M. Basic gait and symmetry measures for primary school-aged children and young adults. II: Walking at slow, free and fast speed. Gait Posture. 2011; 33: 2935. 
Kinematics of Rett walking

552 32. Carriero A, Zavatsky A, Stebbins J, Theologis T, Shefelbine SJ. Correlation between lower

553 limb bone morphology and gait characteristics in children with spastic diplegic cerebral palsy. $J$

554 Pediatr Orthop. 2009; 29: 73-79.

555

556 33. Delabastita T, Desloovere K, Meyns P. Restricted arm swing affects gait stability and

557 increased walking speed alters trunk movements in children with cerebral palsy Front Hum

$558 \quad$ Neurosci. 2016 Jul; 10: 354.

559

560 34. Lelas JL, Merriman GJ, Riley PO, Kerrigan DC, Predicting peak kinematic and kinetic

561 parameters from gait speed. Gait Posture. 2003; 17: 106-112.

562

563 35. Oberg T, Karsznia A, Oberg K. Basic gait parameters: reference data for normal subjects, 10-

56479 years of age. $J$ Rehabil Res Dev. 1993; 30(2): 210-223.

565

566 36. Stoquart G, Detrembleur C, Lejeune T. Effect of speed on kinematic, kinetic,

567 electromyographic and energetic reference values during treadmill walking. Clin Neurophysiol.

$568 \quad 2008 ; 38: 105-116$.

569

570 37. Kutilek P, Viteckova S, Svoboda Z, Smrcka P. Kinematic quantification of gait asymmetry in

571 patients with peroneal nerve palsy based on bilateral cyclograms. J Musculoskelet Neuronal

$572 \quad$ Interact. 2013; 13(2): 244-250.

573 
Kinematics of Rett walking

574 38. Hadizadeh M, Amri S, Mohafez H, Roohi SA, Mokhtar AH. Gait analysis of national

575 athletes after anterior cruciate ligament reconstruction following three stages of rehabilitation

576 program: Symmetrical perspective. Gait Posture. 2016; 48: 152-158.

577

578

39. Nymark JR, Balmer SJ, Melis EH, Lemaire ED, Millar S. Electromyographic and kinematic

579 nondisabled gait differences at extremely slow overground and treadmill walking speeds. J

580 Rehabil Res Dev. 2005; 42(4): 523-534.

581

582

40. Chen G, Patten C, Kothari DH, Zajac FE. Gait differences between individuals with poststroke hemiparesis and non-disabled controls at matched speeds. Gait Posture. 2005; 22: 51-56.

584

585

586

587

588

589

590

591

592

593

594

99-107.

41. Stanhope VA, Knarr BA, Reisman DS, Higginson JS. Frontal plane compensatory strategies associated with self-selected walking speed in individuals post-stroke. Clin Biomech. 2014 May; 29(5): 518-522.

42. Kerrigan DC, Frates EP, Rogan S, Riley PO. Spastic paretic stiff-legged gait: biomechanics of the unaffected limb. Am J Phys Med Rehabil. 1999; 78: 354-360.

43. Larsson G, Julu POO, Engerstrom IW, Sandlund M, Lindstrom B. Walking on treadmill with Rett syndrome-Effects on the autonomic nervous system. Res Dev Disabil. 2018; Dec;83: 
Kinematics of Rett walking

605 606

607 608 609

44. Sutherland DH, Santi M, Abel MF. Treatment of stiff-knee gait in cerebral palsy: a comparison by gait analysis of distal rectus femoris transfer versus proximal rectus release. $J$ Pediatr Orthop. 1990 Jul-Aug; 10(4): 433-441.

45. Kerrigan DC, Gronley J, Perry J. Stiff-legged gait in spastic paresis. A study of quadriceps and hamstrings muscle activity. Am J Phys Med Rehabil. 1991 Dec; 70(6): 294-300.

46. Goldberg SR, Ounpuu S, Delp SL. The importance of swing-phase initial conditions in stiffknee gait. J Biomech. 2003 Aug; 36: 1111-1116.

47. Akalan NE, Kuchimov S, Apti A, Temelli Y, Nene A. Contributors of stiff knee gait pattern for able bodies: Hip and knee velocity reduction and tiptoe gait. Gait Posture. 2016 Jan; 43: 17681.

48. Goldberg SR, Anderson FC, Pandy MG, Delp SL. Muscles that influence knee flexion velocity in double support: implications for stiff-knee gait. J Biomech. 2004 Aug; 37(8): 118996.

49. Akalan NE, Kuchimov S, Apti A, Temelli Y, Nene A. Weakening iliopsoas muscle in healthy adults may induce stiff knee pattern. Acta Orthop Traumatol Turc. 2016; 50: 642-648. 1999; 9: 207-231. 
Kinematics of Rett walking

620

621

622

623

624

625

626

627

628

629

630

631

632

633

634

635

636

637

638

639

640

641

642

643

51. Waters RL, Yakura JS, Adkins R, Barnes G. Determinants of gait performance following spinal cord injury. Arch Phys Med Rehabil. 1989 Nov; 70(12): 811-8.

52. Standsfield BW, Hillman SJ, Hazlewood ME, Lawson AA, Mann AM, Loudon IR, et al.

Normalized Speed, Not Age, Characterizes Ground Reaction Force Patterns in 5- to 12-Year-Old

Children Walking at Self-Selected Speeds. J Pediatr Orthop. 2001; 21: 395-402.

53. Stansfield BW, Hillman SJ, Hazlewood ME, Robb JE. Regression analysis of gait parameters

with speed in normal children walking at self-selected speeds. Gait Posture. 2006; 23: 288-294.

54. Wonsetler EC, Mark G. Bowden MG. A systematic review of mechanisms of gait speed

change post-stroke. Part 2: Exercise capacity, muscle activation, kinetics, and kinematics. Top

Stroke Rehabil. 2017 July; 24(5): 394-403.

55. Tyrell CM, Roos MA, Rudolph KS, Reisman DS. Influence of systematic increases in treadmill walking speed on gait kinematics after stroke. Phys Ther. 2011; 91:391-403.

56. Willerslev-Olsen M, Petersen TH, Farmer SF, Nielsen JB. Gait training facilitates central drive to ankle dorsiflexors in children with cerebral palsy. BRAIN 2015; 138: 589-603.

57. Norton JA, Gorassini MA. Changes in cortically related intermuscular coherence accompanying improvements in locomotor skills in incomplete spinal cord injury. $J$

Neurophysiol. 2006 Apr; 95(4): 2580-2589. 


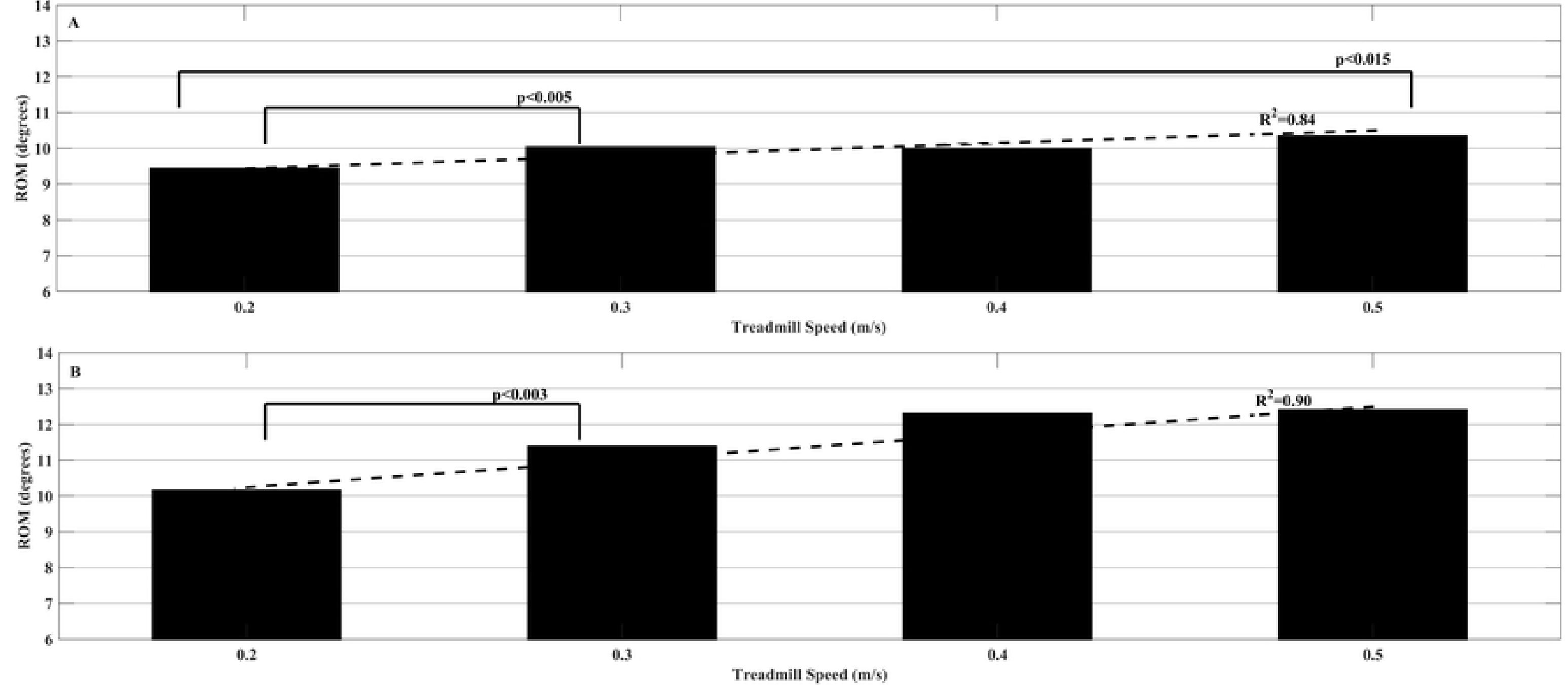

Fig 1 


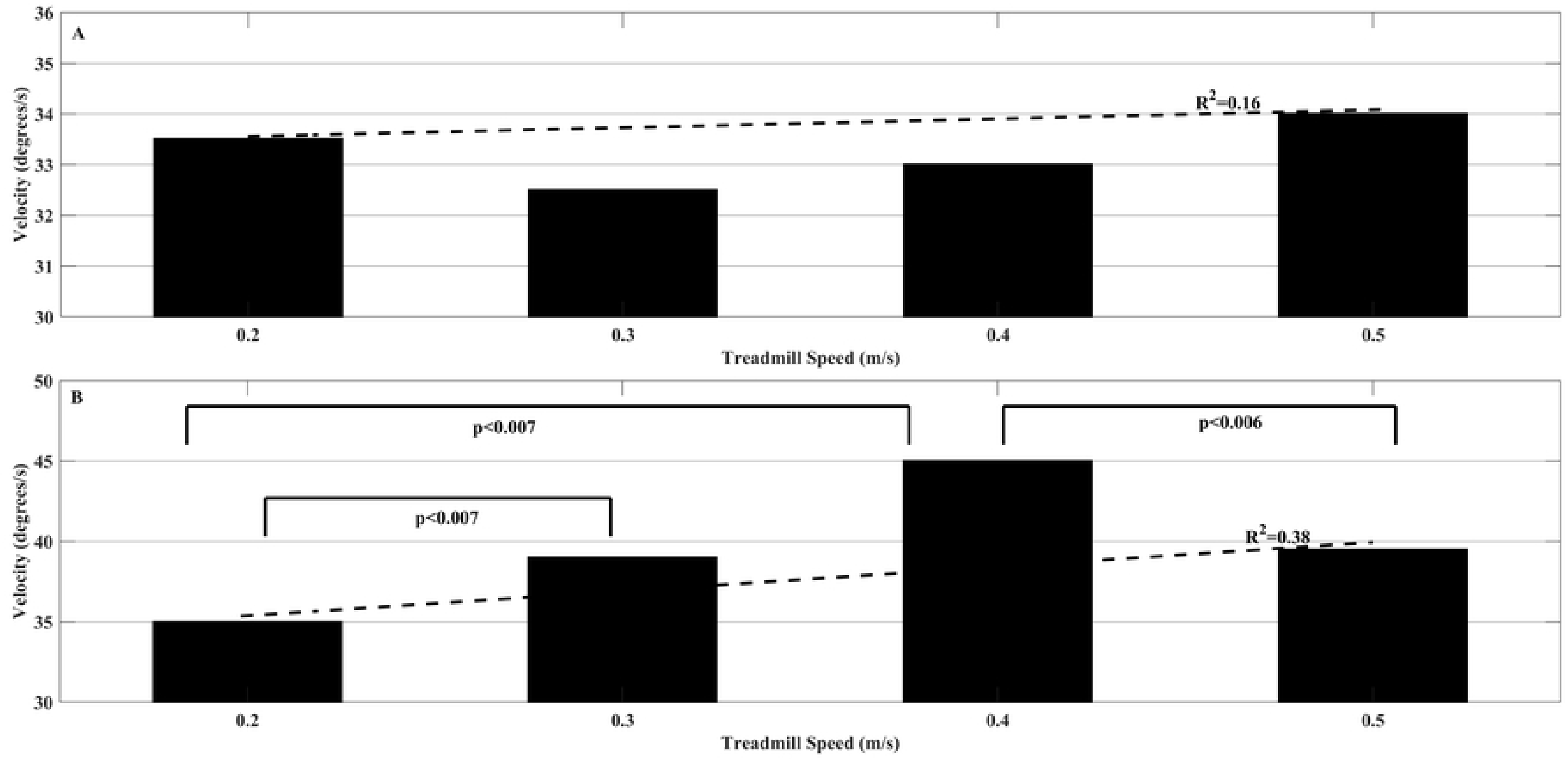

Fig 2 
